\title{
Revisão da distribuição de Suiriri suiriri burmeisteri (Aves: Tyrannidae) no estado de São Paulo, Brasil, com novos registros em seu limite de distribuição sudeste Revision of the distribution of Suiriri suiriri burmeisteri (Aves: Tyrannidae) in the state of São Paulo, Brazil, with new records in its southeastern distribution limits
}

\author{
Arthur Macarrão' (1) | Fernando Igor de Godoy I, II (D) \\ 'Casa da Floresta Ambiental Ltda. Piracicaba, São Paulo, Brasil \\ "Universidade Federal de São Carlos. Programa de Pós-Graduação em Ecologia e Recursos Naturais. São Carlos, São Paulo, Brasil
}

\begin{abstract}
Resumo: O suiriri-cinzento (Suiriri suiriri) apresenta ampla distribuição na América do Sul, estando a subespécie S. s. burmeisteri restrita a fitofisionomias semiabertas dos biomas Cerrado, Caatinga e Pantanal, cuja extensão predomina em território brasileiro. No estado de São Paulo, a escassez de registros e de informações sobre a espécie a levou a ser considerada criticamente ameaçada anteriormente. Aqui, apresentamos novos registros, alguns mais a sudeste do que aqueles conhecidos para o estado, os quais conferem uma pequena expansão de distribuição local. Além disso, revisamos a distribuição da espécie no estado de São Paulo.
\end{abstract}

Palavras-chave: Cerrado. Suiriri-cinzento. Limites de bioma. Mapa de distribuição.

Abstract: The Suiriri Flycatcher (Suiriri suiriri) is widely distributed in South America, with the subspecies S. s. burmeisteri, which occurs mainly in Brazilian territory, restricted to the semi-open phytophysiognomies of the Cerrado, Caatinga, and Pantanal biomes. Due to the scarcity of records and information in the state of São Paulo, the species used to be previously considered as critically endangered. Here we present new records, some of which from further to the southeast than known for this state, and we briefly elaborate on its local distribution. Furthermore, we review the distribution of the species in the state of São Paulo.

Keywords: Cerrado. Suiriri flycatcher. Biome boundaries. Distribution map.

MACARRÃO, A. \& F. I. GODOY, 2020. Revisão da distribuição de Suiriri suiriri burmeisteri (Aves: Tyrannidae) no estado de São Paulo, Brasil, com novos registros em seu limite de distribuição sudeste. Boletim do Museu Paraense Emílio Goeldi. Ciências Naturais 15(2): 475-481. DOI: http://doi.org/10.46357/bcnaturais.v15i2.162.

Autor para correspondência: Arthur Macarrão. Avenida Joaninha Morganti, 289 - Monte Alegre. Piracicaba, SP, Brasil. CEP 13415-030 (arthur@casadafloresta.com.br).

Recebido em 06/08/2019

Aprovado em 08/04/2020

Responsabilidade editorial: Adriano Oliveira Maciel 


\section{INTRODUÇÃO}

O gênero Suiriri possui uma taxonomia complexa, sendo alvo de diversos debates (Hayes, 2001; Zimmer et al., 2001; Lopes \& Marini, 2005; Kirwan et al., 2014). Atualmente, duas espécies são reconhecidas, sendo Suiriri suiriri (Vieillot, 1818)a que apresenta distribuição geográfica mais ampla na América do Sul (Robbins, 2017), com três subespécies assinaladas para o território brasileiro (Piacentini et al., 2015). Suiriri suiriri bahiae é restrita ao Nordeste do Brasil, enquanto S. s. suiriri distribui-se pela Bolívia, pelo Paraguai, pelo Uruguai, pelo Sul do Brasil e pela Argentina, chegando à Patagônia (San Antonio Oeste, Río Negro, Argentina; Llanos et al., 2011). Já a forma conferida para o Brasil central, que ocorre até o oeste do estado de São Paulo, atualmente é tratada como Suiriri suiriri burmeisteri (Kirwan et al., 2014; Piacentini et al., 2015). Esse táxon é aparentemente residente, ao contrário do que é observado para $S$. s. suiriri, que é considerado migrante austral, ou seja, se desloca para regiões do norte de sua distribuição nos meses mais frios (Chesser, 1994; Ridgely \& Tudor, 2009).

No Brasil, a espécie habita o Cerrado, a Caatinga e o Pantanal, além de outras fisionomias abertas de biomas adjacentes, sendo seu habitat característico áreas campestres com árvores esparsas (Alves \& Cavalcanti, 1996; Sick, 1997; Amaral \& Ragusa-Netto, 2008; Uezu, 2009; Olmos, 2009) e também bordas de mata e regiões ecotonais (Hayes, 2001; Sanaiotti \& Cintra, 2001; Ridgely \& Tudor, 2009). Já no estado de São Paulo, onde é considerada quase ameaçada de extinção (São Paulo, 2018), sua distribuição restringe-se a poucos remanescentes, sendo sua sobrevivência afetada pela modificação e supressão de áreas de Cerrado (Uezu, 2009). Entretanto, trata-se de uma espécie com poucas informações a respeito de suas exigências ecológicas, as quais poderiam embasar diretrizes para sua conservação (Uezu, 2009).

Nesse contexto, apresentamos aqui novos registros de Suiriri suiriri no estado de São Paulo. Alguns desses registros são mais a leste do que os já conhecidos, o que confere uma breve expansão de distribuição local. Além disso, foi realizada a revisão dos registros da espécie no estado com base na literatura disponível.

\section{MÉTODOS}

A espécie foi registrada através de excursões a campo no estado de São Paulo, em regiões onde predominam os ecótonos Mata Atlântica-Cerrado. As amostragens foram realizadas entre 2015 e 2017, sempre no período matinal. Vinte e nove fragmentos florestais foram visitados em 24 repetições amostrais. Os dois pesquisadores utilizaram binóculos Bushnell $10 \times 42 \mathrm{~mm}$ para visualização, câmeras fotográficas Nikon P520 e Canon EOS 7D (acoplada de lente 100-400 mm) e um gravador digital Tascam DR 100 com microfone unidirecional Rode NTG02, para documentação.

Para dados secundários, foram consultados os trabalhos de Willis \& Oniki (2003) e Uezu (2009), que englobam dados históricos, além da base de dados online do WikiAves (2019) e a do Xeno-canto (2019). Foram também consultadas coleções biológicas, tais como as inseridas na plataforma SpeciesLink (2019), no Museu de Zoologia da Universidade Estadual de Campinas (ZUEC), no American Museum of Natural History (AMNH), no Florida Museum of Natural History (FLMNH) e no National Museum of Natural History (NMNH), da Smithsonian Institution, Washington, além da compilação de Pinto (1944) e Hasui et al. (2018). Somente foram considerados dados dos acervos online quando corretamente identificados, uma vez que essas bases são públicas e estão sujeitas a inserções de registros equivocados. Por isso, dados como os obtidos na base e-bird foram desconsiderados, devido à impossibilidade de checar registros duvidosos.

\section{RESULTADOS}

De acordo com os dados obtidos em campo, a espécie foi registrada em dez localidades, correspondentes a sete municípios do estado de São Paulo (Figura 1, Tabela 1). Os registros mais a leste ocorreram em Campinas (Figura 2A) (Macarrão, 2015), em remanescentes de Cerrado, de

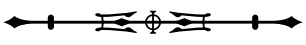




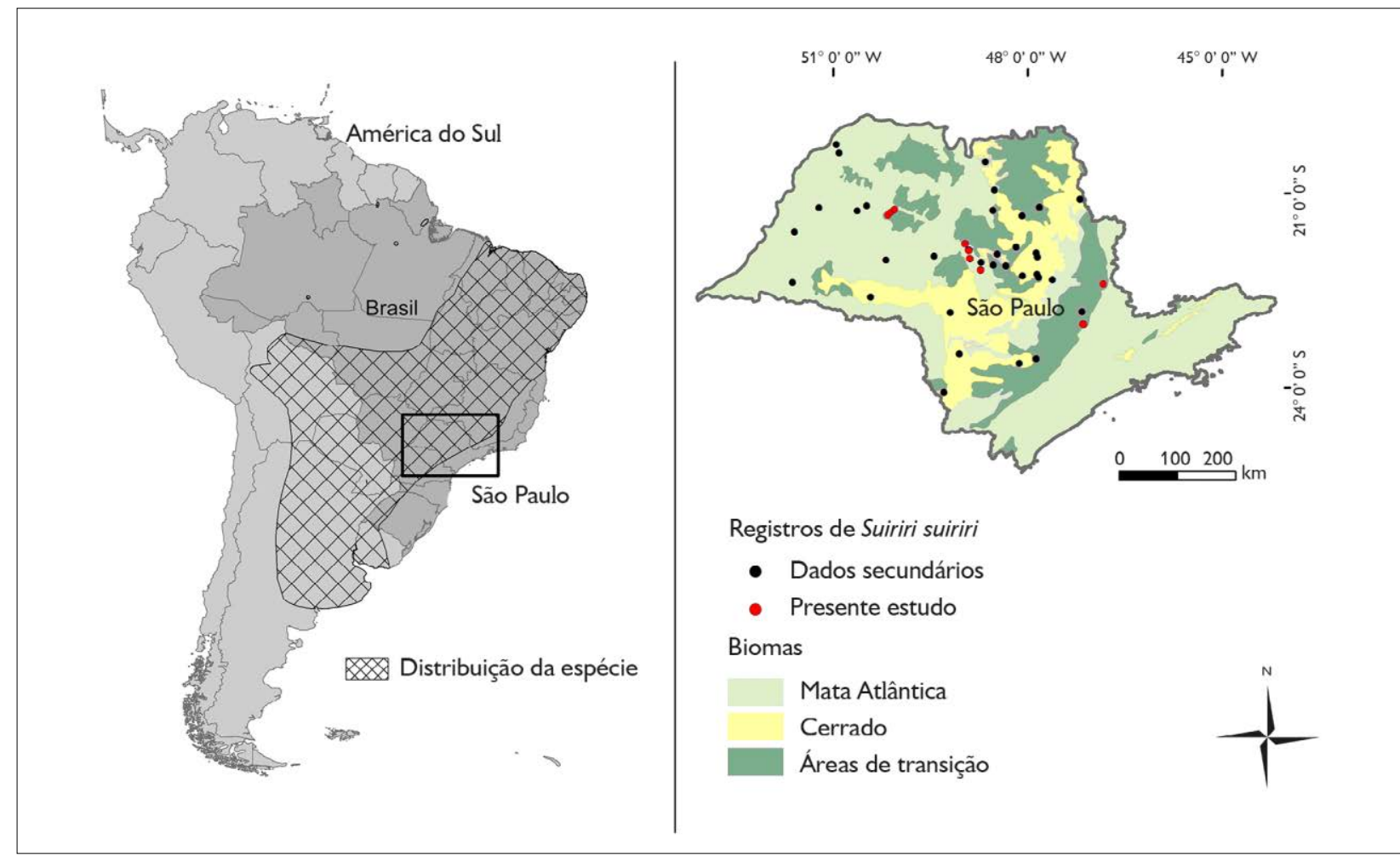

Figura 1. Registros do suiriri-cinzento (Suiriri suiriri burmeisteri) no estado de São Paulo. Pontos vermelhos correspondem ao presente estudo e pontos cinzas, a dados secundários (biomas estão de acordo com IBGE, 2004).

propriedade do aeroporto de Viracopos. Foram anotados três pontos de ocorrência, dois deles sendo de dois indivíduos, em uma área aberta com árvores esparsas. Os registros foram realizados nas datas 23/01/2015 e 13/02/2015. Essa área, hoje, encontra-se bastante modificada com as obras do aeroporto. A vegetação consiste em um remanescente de Cerrado antropizado, com o solo coberto por braquiária (Urochloa sp.). Outro ponto apresentou somente um indivíduo, no dia 10/07/2015, no interior de uma área de cerradão, um ambiente dominado por árvores de grande porte da família Fabaceae.

Os outros registros foram feitos em bordas de florestas (floresta estacional semidecídua ou cerradão), com diferentes estados de conservação e sucessão, com predominância em estágios sucessionais inicial e intermediário, todos imersos em matriz de cana-de-açúcar
Tabela 1. Localidades dos novos registros de Suiriri suiriri no estado de São Paulo.

\begin{tabular}{c|c|c}
\hline Município & \multicolumn{2}{|c}{ Coordenadas } \\
\hline Campinas & $23^{\circ} 1^{\prime} 4,35^{\prime \prime} \mathrm{S}$ & $47^{\circ} 7^{\prime} 44,91^{\prime \prime} \mathrm{O}$ \\
\hline Campinas & $23^{\circ} 0^{\prime} 47,27^{\prime \prime} \mathrm{S}$ & $47^{\circ} 8^{\prime} 50,92^{\prime \prime} \mathrm{O}$ \\
\hline Itapira & $22^{\circ} 23^{\prime} 53,64^{\prime \prime} \mathrm{S}$ & $46^{\circ} 49^{\prime} 56,03^{\prime \prime} \mathrm{O}$ \\
\hline Boraceia & $22^{\circ} 10^{\prime} 40,56^{\prime \prime} \mathrm{S}$ & $48^{\circ} 43^{\prime} 29,82^{\prime \prime} \mathrm{O}$ \\
\hline Arealva & $22^{\circ} 0^{\prime} 16,08^{\prime \prime} \mathrm{S}$ & $48^{\circ} 53^{\prime} 36,07^{\prime \prime} \mathrm{O}$ \\
\hline Iacanga & $21^{\circ} 46^{\prime} 40,48^{\prime \prime} \mathrm{S}$ & $48^{\circ} 57^{\prime} 58,56^{\prime \prime} \mathrm{O}$ \\
\hline Iacanga & $21^{\circ} 52^{\prime} 34,69^{\prime \prime} \mathrm{S}$ & $48^{\circ} 54^{\prime} 32,53^{\prime \prime} \mathrm{O}$ \\
\hline Barbosa & $21^{\circ} 14^{\prime} 44,88^{\prime \prime} \mathrm{S}$ & $50^{\circ} 3^{\prime} 23,65^{\prime \prime} \mathrm{O}$ \\
\hline Penápolis & $21^{\circ} 17^{\prime} 34,31^{\prime \prime} \mathrm{S}$ & $50^{\circ} 6^{\prime} 53,58^{\prime \prime} \mathrm{O}$ \\
\hline Penápolis & $21^{\circ} 20^{\prime} 4,19^{\prime \prime} \mathrm{S}$ & $50^{\circ} 9^{\prime} 31,06^{\prime \prime} \mathrm{O}$ \\
\hline
\end{tabular}

ou pastagens (Figuras 2 e 3) (Macarrão, 2016; Godoy, 2017a, 2017b). Um único registro foi realizado em mata ciliar, no município de Itapira.

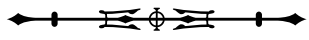




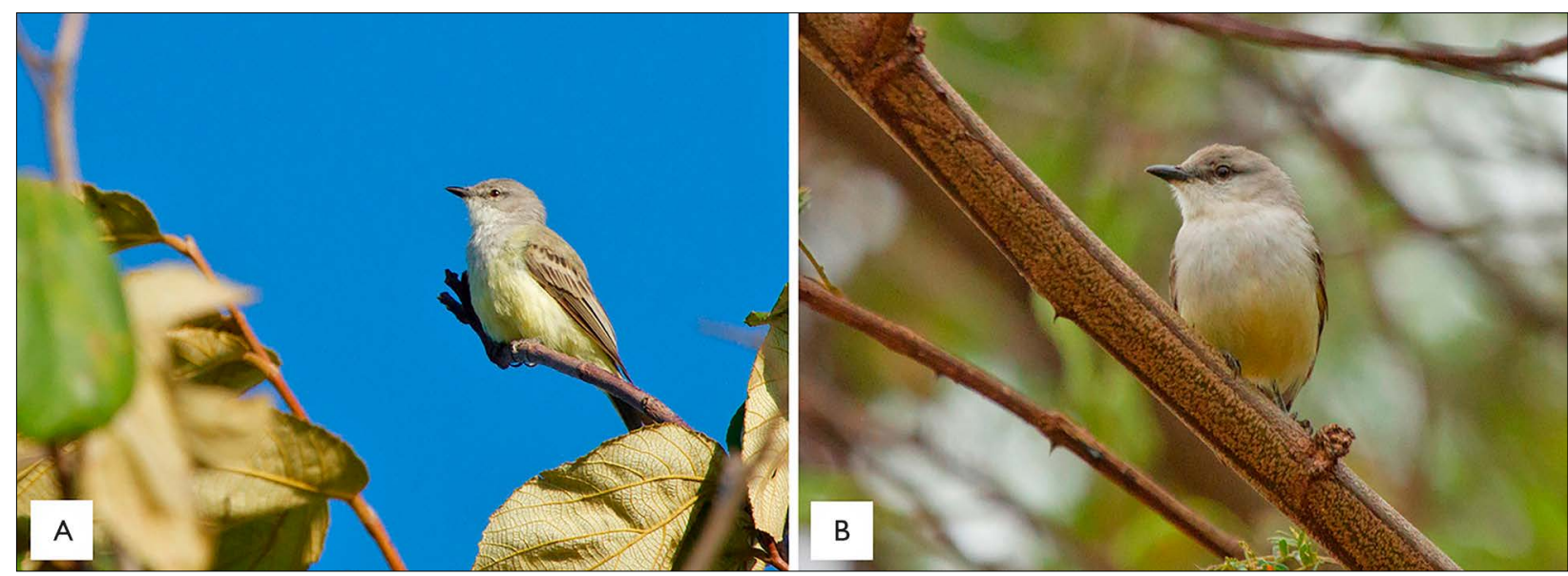

Figura 2. Suiriri suiriri burmeisteri fotografado em Campinas, São Paulo, em 2015 (A), e Penápolis, São Paulo, em 2016 (B). Fotos: Arthur Macarrão.
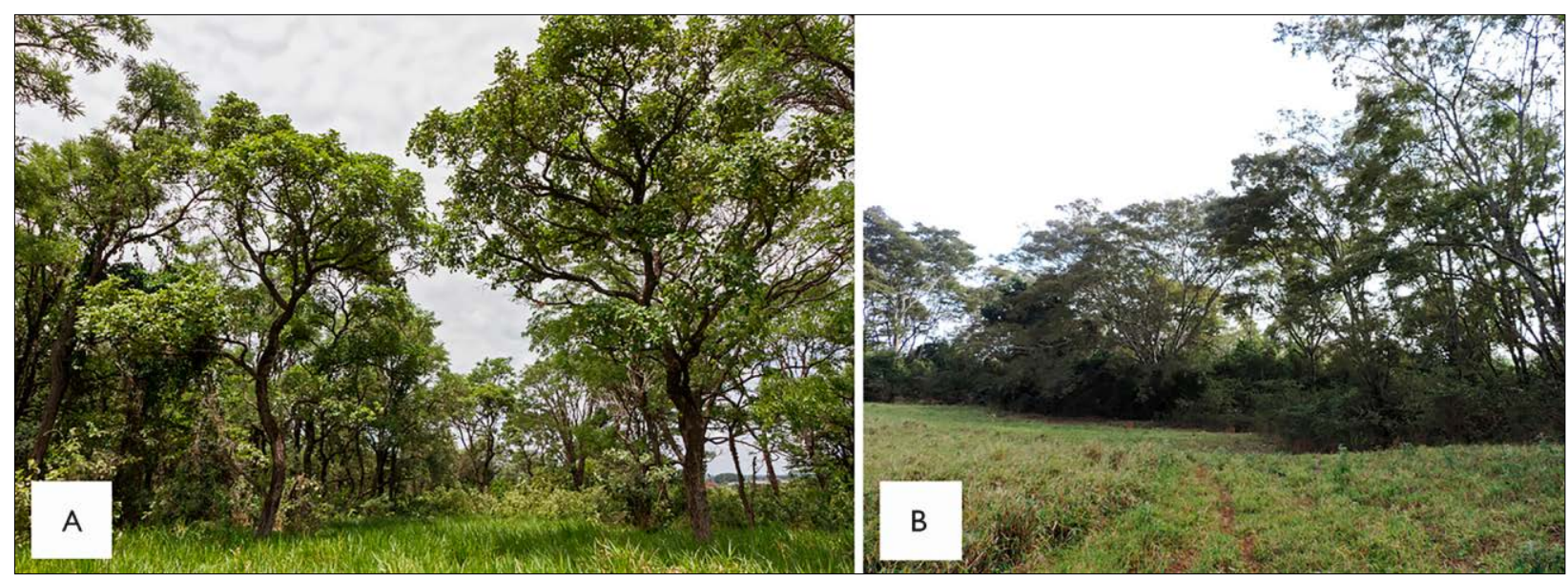

Figura 3. Ambientes de registro de Suiriri suiriri burmeisteri em Campinas, São Paulo, em 2015 (A); e lacanga, São Paulo, em 2016 (B). Fotos: Arthur Macarrão.

A revisão de dados de literatura apontou registros para mais 33 localidades, com concentração em áreas de Cerrado e transição no centro do estado (Figura 1). Aleixo \& Vielliard (1995) não registraram a espécie em levantamentos realizados na Mata de Santa Genebra, um fragmento de 251 hectares em Campinas, localidade a aproximadamente 21 quilômetros dos nossos registros. Possivelmente, a espécie não ocorre nessa Unidade de Conservação por conta da fitofisionomia predominante na área, que se trata de uma floresta estacional semidecídua, ambiente mais fechado do que o utilizado pela espécie.

\section{DISCUSSÃO}

O fato de a maioria dos registros ter sido efetuada em locais com alterações antrópicas (Figura 3) sugere que a espécie não seja tão exigente em relação ao habitat. Todavia, não é sabido ao certo qual o tempo de permanência da espécie em ambientes alterados, a qual pode apenas utilizar tais áreas ocasionalmente, o que também dificultaria sua detecção. De acordo com Zimmer et al. (2001), S. suiriri tem persistido em áreas que sofreram queimadas intensas, embora se acredite que repetições do fogo possam afetá-la. Isso foi observado por Cavalcanti \& Alves (1997), os quais

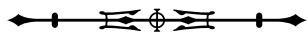


não notaram alterações comportamentais evidentes da espécie com as queimadas.

A preferência por ambientes com heterogeneidade estrutural, ou seja, mosaicos de áreas semiabertas, florestadas e campestres, pode estar relacionada ao hábito reprodutivo, já que, embora a espécie seja comum em áreas savânicas (Cavalcanti \& Alves, 1997), aparenta priorizar indivíduos arbóreos para nidificação (Lopes \& Marini, 2005). Além disso, a copa das árvores constitui um dos principais ambientes de forrageio (Cavalcanti \& Alves, 1997), fatores que justificariam sua presença em bordas florestais. De acordo com Lopes \& Marini (2006), a espécie parece evitar áreas extremamente abertas, sendo encontrada em fisionomias mais densas do Cerrado.

Willis \& Oniki (2003) apontaram a ocorrência da espécie em três municípios limítrofes, entre os biomas Cerrado e Mata Atlântica, no estado de São Paulo: Itararé, Itapetininga e Alambari. Contudo, os registros apresentados neste estudo para a região de Campinas constituem seu limite leste de distribuição no estado. Além disso, um espécime depositado no ZUEC foi coletado no município de Sumaré em 1988, distante apenas cerca de $30 \mathrm{~km}$ da localidade. Desse modo, todos esses registros acompanham a possível linha divisória entre os biomas determinada pelo IBGE (2004), limitando-se ao perímetro do Cerrado (Figura 1). Essa concentração de pontos em áreas de Cerrado ou de transição evidencia a relação do táxon S. s. burmeisteri com esse bioma e fisionomias correspondentes.

Os registros aqui apresentados estão de acordo com Sick (1997) e Olmos (2009) no que se refere ao habitat da espécie. Tais autores afirmam que a espécie habita o 'espinilho', termo normalmente atribuído a diversos tipos de leguminosas arbustivas e arbóreas, com folhas compostas bipinadas. Lopes \& Marini (2005) indicam que a maioria dos ninhos encontrados em seu estudo foi nesse tipo de vegetação, no caso, em árvores Dimorphandra mollis Benth. e Stryphnodendron adstringens (Mart.) Coville.
Até meados de 2018, a espécie era incluída em uma categoria de ameaça regional, o que podia estar atrelado à subestimação de registros, e não necessariamente à perda do Cerrado, já que a espécie ocupa também áreas perturbadas. Essa escassez de informações também estaria relacionada ao fato de que poucos pesquisadores e observadores de aves visitavam o oeste e centro do estado até alguns anos, visto que recentemente têm aumentado os registros na região (WikiAves, 2019). Além disso, a espécie também possui plumagem críptica, semelhante a outras espécies da família, o que a torna de difícil diagnose visual.

Em decorrência dessas dúvidas, a espécie ainda é merecedora de atenção no estado de São Paulo, de modo a ser avaliada quanto a parâmetros demográficos. Os registros aqui apresentados são de suma importância para ampliar o conhecimento sobre Suiriri suiriri, bem como para auxiliar a análise de seu status localmente, já que seus registros predominam em áreas alteradas. Nesse contexto, ressalta-se a importância de maior atenção à presença da espécie em fisionomias vegetacionais semiabertas, incluindo ambientes antropizados, uma vez que pode passar despercebida.

\section{AGRADECIMENTOS}

Esse trabalho foi apoiado pela AES Tietê Energia S.A., como parte do programa de monitoramento intitulado "Programa de conservação e monitoramento da fauna terrestre", e foi desenvolvido pela Casa da Floresta Ambiental SS. Somos gratos a Tatiane Rech e Odemberg Veronez, por providenciarem todo suporte operacional. A Janderson Kauan Felipe e Leandro Morais da Silva, pelo o apoio logístico nos trabalhos de campo, a Rodrigo Bernardo, por fazer os mapas, a Vagner de A. Gabriel, pela revisão do material, a Rogério Cânovas, por ajudar na identificação de plantas, e a Marcelo Ducatti, pela coordenação do monitoramento de fauna. Os autores também agradecem aos revisores pelas valiosas contribuições e apontamentos ao manuscrito.

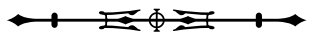




\section{REFERÊNCIAS}

ALEIXO, A. \& J. M. E. VIELLIARD, 1995. Composição e dinâmica da avifauna da mata de Santa Genebra, Campinas, São Paulo, Brasil. Revista Brasileira de Zoologia 12(3): 493-511. DOI: https://doi.org/10.1590/S0101-81751995000300004

ALVES, M. A. S. \& R. B. CAVALCANTI, 1996. Sentinel behavior, seasonality, and the structure of bird flocks in a Brazilian savanna. Ornitologia Neotropical 7: 43-51.

AMARAL, P. P. \& J. RAGUSA-NETTO, 2008. Bird mixed-flocks and nuclear species in a tecoma savanna in the Pantanal. Brazilian Journal of Biology 68(3): 511-518. DOI: https://doi.org/10.1590/ S1519-69842008000300007

CAVALCANTI, R. B. \& M. A. S. ALVES, 1997. Effects of fire on savanna birds in central Brazil. Ornitologia Neotropical 8(1): 85-87.

CHESSER, R. T., 1994. Migration in South America: an overview of the austral system. Bird Conservation International 4(2-3): 91-107. DOI: https://doi.org/10.1017/S0959270900002690

GODOY, F. I., 2017a. WA3716227, Suiriri suiriri (Vieillot, 1818). In: WikiAves - A Enciclopédia das Aves do Brasil. Disponível em: http://www.wikiaves.com/3716227. Acesso em: 11 março 2020.

GODOY, F. I., 2017b. XC533825 - suiriri-cinzento - Suiriri suiriri burmeisteri. In: Xeno-Canto. Disponível em: https://www.xenocanto.org/533825. Acesso em: 11 março 2020.

HASUI, É., J. P. METZGER, R. G. PIMENTEL, L. F. SILVEIRA, A. A. A. BOVO, A. C. MARTENSEN, A. UEZU, A. L. REGOLIN, A. A. B. OLIVEIRA, C. A. F. R. GATTO, C. DUCA, C. B. ANDRETTI, C. BANKS-LEITE, D. LUZ, D. MARIZ, E. R. ALEXANDRINO, F. M. BARROS, F. MARTELLO, I. M. S. PEREIRA, J. N. SILVA, K. M. P. M. B. FERRAZ, L. N. NAKA, L. ANJOS, M. A. EFE, M. A. PIZO, M. PICHORIM, M. S. S., GONÇALVES, P. H. C. CORDEIRO, R. A. DIAS, R. L. MUYLAERT, R. C. RODRIGUES, T. V. V. COSTA, V. CAVARZERE, V. R. TONETTI, W. R. SILVA, C. N. JENKINS, M. GALETTI \& M. C. RIBEIRO, 2018. ATLANTIC BIRDS: a data set of bird species from the Brazilian Atlantic Forest. Ecology 99(2): 497. DOI: https://doi.org/10.1002/ecy.2119

HAYES, F. E., 2001. Geographic variation, hybridization, and the leapfrog pattern of evolution in the Suiriri flycatcher (Suiriri Suiriri) complex. The Auk 118(2): 457-471. DOI: https://doi. org/10.1642/0004-8038(2001)118[0457:GVHATL]2.0.CO;2

INSTITUTO BRASILEIRO DE GEOGRAFIA E ESTATÍSTICA (IBGE), 2004. Mapa de vegetação do Brasil. Disponível em: https:// ww2.ibge.gov.br/home/presidencia/noticias/21052004biomas. shtm/. Acesso em: 20 abril 2019.
KIRWAN, G. M., F. D. STEINHEIMER, M. A. RAPOSO \& K. J. ZIMMER, 2014. Nomenclatural corrections, neotype designation and new subspecies description in the genus Suiriri (Aves: Passeriformes: Tyrannidae). Zootaxa 3784(3): 224-240. DOI: https://doi. org/10.11646/zootaxa.3784.3.2

LLANOS, F. A., M. FAILLA, G. J. GARCÍA, P. M. GIOVINE, M. CARBAJAL, P. M. GONZÁLEZ, D. P. BARRETO, P. QUILLFELDT \& J. F. MASELLO, 2011. Birds from the endangered Monte, the Steppes and Coastal biomes of the province of Río Negro, northern Patagonia, Argentina. Check List 7(6): 782-797. DOI: https://doi. org/10.15560/11025

LOPES, L. E. \& M. Â. MARINI, 2005. Biologia reprodutiva de Suiriri affinis e S. islerorum (Aves: Tyrannidae) no cerrado do Brasil central. Papéis Avulsos de Zoologia 45(12): 127-141. DOI: https://doi. org/10.1590/S0031-10492005001200001

LOPES, L. E. \& M. Â. MARINI, 2006. Home range and habitat use by Suiriri affinis and Suiriri islerorum (Aves: Tyrannidae) in the central Brazilian Cerrado. Studies on Neotropical Fauna and Environment 41(2): 87-92. DOI: https://doi.org/10.1080/01650520500309826

MACARRÃO, A., 2015. WA3716275, Suiriri suiriri (Vieillot, 1818). In: WikiAves - A Enciclopédia das Aves do Brasil. Disponível em: http://www.wikiaves.com/3716275. Acesso em: 11 março 2020.

MACARRÃO, A., 2016. WA3717264, Suiriri suiriri (Vieillot, 1818). In: WikiAves - A Enciclopédia das Aves do Brasil. Disponível em: http://www.wikiaves.com/3717264. Acesso em: 11 março 2020.

OLMOS, A., 2009. Aves en el Uruguay. Trandinco, Montevideo.

PIACENTINI, V. Q., A. ALEIXO, C. E. AGNE, G. N. MAURÍCIO, J. F. PACHECO, G. A. BRAVO, G. R. R. BRITO, L. N. NAKA, F. OLMOS, S. POSSO, L. F. SILVEIRA, G. S. BETINI, E. CARRANO, I. FRANZ, A. C. LEES, L. M. LIMA, D. PIOLI, F. SCHUNCK, F. R. AMARAL, G. A. BENCKE, M. COHN-HAFT, L. F. A. FIGUEIREDO, F. C. STRAUBE \& E. CESARI, 2015. Annotated checklist of the birds of Brazil by the Brazilian Ornithological Records Committee. Revista Brasileira de Ornitologia 23(2): 91-298.

PINTO, O. M. O., 1944. Catálogo das aves do Brasil e lista dos exemplares existentes na coleção do Departamento de Zoologia. Departamento de Zoologia, Secretaria da Agricultura, Indústria e Comércio, São Paulo.

RIDGELY, R. S. \& G. TUDOR, 2009. Field guide to the songbirds of South America: the passerines. University of Texas Press, Austin.

ROBBINS, M., 2017. Suiriri flycatcher (Suiriri suiriri). In: J. DEL HOYO, A. ELLIOTT, J. SARGATAL, D. A. CHRISTIE \& E. DE JUANA (Ed.): Handbook of the birds of the world alive. Lynx Edicions, Barcelona. Disponível em: http://www.hbw.com/node/57164. Acesso em: 12 dezembro 2017.

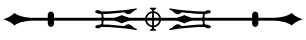


SANAIOTTI, T. M. \& R. CINTRA, 2001. Breeding and migrating birds in an Amazonian Savanna. Studies on Neotropical Fauna and Environment 36(1): 23-32. DOI: http://doi.org/10.1076/ snfe.36.1.23.8878

SÃO PAULO (Estado). Decreto n. 63.853 de 27 de novembro de 2018. Declara as espécies da fauna silvestre no Estado de São Paulo regionalmente extintas, as ameaçadas de extinção, as quase ameaçadas e as com dados insuficientes para avaliação, e dá providências correlatas. Diário Oficial do Estado de São Paulo 128(221): 25. Seção 1.

SICK, H., 1997. Ornitologia brasileira. Nova Fronteira, Rio de Janeiro.

SPECIESLINK, 2019. Sistema de informação distribuído para coleções biológicas. Disponível em: http://splink.cria.org.br/. Accesso em: 27 julho 2019.
UEZU, A., 2009. Suiriri suiriri (Vieillot, 1818). Passeriformes, Tyrannidae. In: P. M. BRESSAN, M. C. KIERULFF \& A. M. SUGIEDA (Ed.): Fauna ameaçada de extinção no estado de São Paulo: vertebrados: 220. Fundação Parque Zoológico de São Paulo/ Secretaria do Meio Ambiente, São Paulo.

WILLIS, E. O. \& Y. ONIKI, 2003. Aves do estado de São Paulo. Divisa, Rio Claro.

WIKIAVES, 2019. A enciclopédia das aves do Brasil. Disponível em: http://www.wikiaves.com.br/. Acesso em: 20 julho 2019.

XENO-CANTO, 2019. Xeno-canto: sharing bird sounds from around the world. Disponível em: http://www.xeno-canto.org/. Acesso em: 27 julho 2019.

ZIMMER, K. J., A. WHITTAKER \& D. C. A. OREN, 2001. Cryptic new species of flycatcher (Tyrannidae: Suiriri) from the Cerrado region of Central South America. The Auk 118(1): 56-78. 
